\title{
Aegeline vs Statin in the treatment of Hypercholesterolemia: A comprehensive study in rat model of liver steatosis
}

\author{
A. Singh ${ }^{1}$, S. Gowtham ${ }^{1}$, L.N. Chakrapani ${ }^{1}$, S. Ashokkumar ${ }^{1}$, S.N. Kishore Kumar ${ }^{1}$, V. \\ Prema $^{1}$, R. Divya Bhavani ${ }^{1}$, T. Mohan ${ }^{1}$, Y. K. Sathyamoorthy ${ }^{2}$ \\ ${ }^{1}$ Department of Medical Biochemistry, University of Madras, IBMS, Taramani Campus, \\ Chennai, 600113, Tamilnadu, India; ${ }^{2}$ Department of Anatomy University of Madras, IBMS \\ Taramani, Chennai 600113, Tamilnadu, India
}

Corresponding Author: Periandavan Kalaiselvi, Ph.D., Department of Medical Biochemistry, University of Madras, IBMS, Taramani Campus, Chennai 600113, Tamilnadu, India

Submission Date: August $2^{\text {nd }}, 2017$, Acceptance Date: December $27^{\text {th }}$, 2017, Publication Date: January $31^{\text {st }}, 2018$

Citation: Singh A., Gowtham S., Chakrapani L.N., Ashokkumar S., Kumar S.N., Prema V., Bhavani R., Mohan T., Sathyamoorthy Y.K., Aegeline vs Statin in the treatment of Hypercholesterolemia: A comprehensive study in rat model of liver steatosis. Functional Foods in Health and Disease 2018; 8(1): 1-16. DOI: https://doi.org/10.31989/ffhd.v8i1.381

\begin{abstract}
Background: Aegeline (AG) is a natural hydroxyamide which has not been explored in depth for its potential applications in food, pharmaceutical, and cosmetic industries. Accordingly, in this study, the property of AG as antioxidant, anti-hypercholesterolemic, and an anti-aging component was explored. The hepatoprotective effect of AG on hypercholesterolemic rats was investigated for health care benefits as a hypocholesterolemic drug vs Atorvastatin (AV) for the first time.
\end{abstract}

Objective: The objective was to delineate the mechanism of action of AG in impeding high cholesterol diet (HCD) induced hepatic steatosis in a rat model with special reference to antioxidant status and expression of Nuclear Factor Kappa B (NF-kB) mediated cell adhesion molecules.

Methods: Thirty-six Wistar rats were divided into six groups. The pathology group rats were fed with HCD for 16 weeks and treatment groups rats were fed with HCD for 12 weeks and further supplemented with AG/AV for another 4 weeks. In vivo and in vitro experiments were carried out to find out the relative markers of the pathological condition likely Malondialdehyde (MDA), Oxidized LDL (Ox-LDL), and CRP levels were determined. Standard biochemical parameters were carried out. The expression profile by Western blot analysis was performed to assess the levels of NF- $\mathrm{BB}$, Interleukin 6(IL6) and vascular cell adhesion molecule1 (VCAM1), in the 
hepatic tissues of various experimental groups. Additionally, histopathological examination of liver tissue sections was carried out.

Results: AG administration effectively decreased the oxidative stress induced by HCD as evidenced by MDA levels. Furthermore, the lowered levels of Ox-LDL and CRP in AG administered rats deem it to be a potent anti-inflammatory agent. Compared to AV, AG had a pronounced effect in down-regulating the expression of cell adhesion molecules.

Conclusion: Thus, this study validates $A G$ to be an effective stratagem in bringing down the oxidative stress induced by HCD and can be deemed as an anti-hypercholesterolemic and antisteatotic agent.

Keywords: Aegeline, Atorvastatin, liver, hypercholesterolemia, aging

\section{INTRODUCTION}

Various aromatic compounds in plants that form an indispensable part of the human diet contain antioxidant properties. The antioxidant property of these phenolic compounds, which are mostly flavonoids, depends on their structure and position of the hydroxyl groups. The by-products of such flavonoids have been the subject of investigations and have also proven to be an effective source of phenolic antioxidants. Natural therapeutics such as polyphenols and amides have generated significant interest for the impediment of diseases including cancers, cardiovascular diseases, and degenerative diseases. One such compound of interest is "AG" which is obtained from the plant Aegle marmelos belonging to the family Rutaceae. The ancient systems of medicine including Roman, Ayurveda, Greek, Siddha, and Unani have mentioned therapeutic applications of plant Aegle marmelos in cardiovascular disorders, diabetes, diarrhea, and dysentery [27]. Several phytochemicals like AG, alloimkperatorin, marmelide, marmeline, marmelosin, marmesin, proralem, and tannic acid have been reported to be present in Aegle marmelos [19]. The lipid-lowering activity of ethanolic extract of Aegle marmelos leaves in hyperlipidaemic models of Wistar albino rats has been established [32]. In particular, AG the major phytochemical present in the leaf extract of Aegle marmelos, is a compound which shows structural similarity to the adrenergic receptor agonist that acts as a major lipolytic agent. However, its potential as an efficient atheroprotective agent has not been deeply explored.

\section{MATERIALS AND METHODS}

\section{Drugs and Chemicals:}

AG was procured from Clearsynth Canada. Bovine Serum Albumin (BSA) was procured from Sigma-Aldrich, USA. All other chemicals used were of the analytical grade and were obtained from Medox Biotech India, SRL (Sisco Research Laboratories Pvt. Ltd), Genei, Bangalore and Central Drug House Pvt. Ltd (CDH) Mumbai India). The standard diet was procured from Hindustan Lever Ltd., under commercial name "GOLD MOHUR RAT FEED” containing 5\% fat, $21 \%$ protein, $55 \%$ nitrogen-free extract, $4 \%$ fiber $(\mathrm{w} / \mathrm{w})$ with adequate vitamins and minerals. 


\section{Experimental animals:}

Healthy male albino rats of Wistar strain (n=36; weight, 130-250 g), 12 weeks old (Young rats) and 120 weeks old (Aged rats) procured from Animal House Facility Taramani Campus University of Madras were housed in polypropylene cages, maintained under standardized conditions (12 $\mathrm{h}$ light/dark cycles, $28 \pm 2^{\circ}$ ) with paddy husk bedding at the central animal house, and had access to food and water ad libitum. All experiments were approved by the Institutional Animal Ethical Committee of The University of Madras, Taramani Campus (IAEC No \#01/19/13).

\section{Experimental Cell Line:}

The monolayer cultures of macrophage infected cell line passage number 21 (IC-21 cells) was obtained from NCCS, Pune, India. Cells were cultured in RPMI 1640 with ATCC modification supplemented with 10\% heat-inactivated fetal bovine serum (FBS), Antibiotics (mixture 1\% penicillin/streptomycin/nystatin). Cells were incubated in $\mathrm{T} 25$ tissue culture flasks at $37^{\circ} \mathrm{C}$ in a humidified atmosphere (5\% CO2 \& 95\% air environment). AG was dissolved in $0.1 \%$ DMSO and Ox-LDL were suspended in PBS. AG at the concentration of $10 \mu \mathrm{M}$ and $\mathrm{AV}$ at the concentration of $10 \mu \mathrm{M}$ dissolved in $0.1 \%$ DMSO were used for studies. Human native LDL was prepared from the serum of fasted normolipidemic volunteers. The LDL was precipitated by adding an equal volume of serum and buffered temperature. Centrifuged at $9500 \mathrm{X} \mathrm{g}$ for 5 minutes and the precipitate was suspended in PBS ( $\mathrm{pH}$. 7.4). The LDL was oxidized at $37^{\circ} \mathrm{C}$ for 24 hours by incubating with $5 \mu \mathrm{M} \mathrm{CuSO} 4$ and sterilized by filtration through $0.22 \mu \mathrm{m}$ filter Steinbrecher et al. [33]. The cells were then divided into five groups for various experiments to be performed (Group 1 consisted of control cells, Group 2 consisted cells treated with Ox-LDL, Group 3 consisted cells treated with Ox-LDL +AG, Group 4 consisted cells treated with Ox-LDL $+\mathrm{AV}$, Group 5 consisted of control cells $+\mathrm{AG}$ ).

\section{Experimental Design:}

Hypercholesterolemia was induced by HCD consisting of the standard rat feed (commercial rat feed containing 5\% fat, $21 \%$ protein, $55 \%$ nitrogen-free extract, $4 \%$ fiber (w/w) with adequate vitamins and minerals) supplemented with $4 \%$ cholesterol and $1 \%$ cholic acid [31]. Dose fixation studies were performed for $\mathrm{AG}$, different concentrations ranging from $10 \mathrm{mg}$ to $50 \mathrm{mg}$ were administered for 30 days to aged hyperlipidaemic rats. The optimal dose of AG was found to be 20 $\mathrm{mg} / \mathrm{kg}$ body weight, based on its potential effect to reduce lipid profile, to scavenge free radicals and to boost antioxidant status (Data not included). The experimental animals were grouped as the following: 1) Young control group $(n=6)$, where the young rats (12 weeks old) were fed with a standard rat feed (commercial rat feed containing 5\% fat, $21 \%$ protein, 55\% nitrogen free extract, $4 \%$ fibre (w/w) with adequate vitamins and minerals) throughout the experimental period; 2) Aged control group $(n=6)$, where the aged rats were fed with a standard rat feed throughout the experimental period 3) HCD induced aging rats $(n=6)$, where the aged animals were fed with HCD throughout the experimental period; 4) Treatment group; Aged HCD rats treated with AG (HCD+AG $20 \mathrm{mg} / \mathrm{kg}$ b.w, n=6), in which the animals were fed HCD diet for 60 days, followed by HCD with oral administration of AG for the last 30 days; 5) Standard drug-treated group; Aged HCD rats, treated with AV (HCD+AV $1.5 \mathrm{mg} / \mathrm{kg}$ b.w, n=6), where the animals were fed HCD diet for 60 days, followed by HCD with oral administration of AV for the last 30 days; 6) Drug control 
group; Aged rats were fed standard rat feed for 60 days followed by standard rat feed with oral administration of $\mathrm{AG}$ for the last 30 days.

\section{Sample preparation}

At the end of the experimental period, rats were anesthetized with ketamine $(22 \mathrm{mg} \mathrm{kg}-1, \mathrm{i} / \mathrm{m})$ and blood samples were collected via tail vein into anticoagulant-containing and anticoagulantfree test tubes. The liver was collected immediately, immersed in ice-cold physiological saline, and weighed. Small sections from each tissue were kept aside for histological studies. A $10 \%$ tissue homogenate was prepared using Tris- $\mathrm{HCl}$ buffer $(0.01 \mathrm{M}, \mathrm{pH} 7.4)$, followed by centrifugation at $12,000 \mathrm{rpm}$ for $10 \mathrm{~min}$. The supernatant was used for the analysis of various parameters. The rest of the tissue was stored at $-80{ }^{\circ} \mathrm{C}$ for protein expression studies. The protein concentration of the tissue homogenate was determined by the standard method of Lowry et al. [18] using bovine serum albumin as a standard.

\section{Body Weight Gain (BWG)}

Body weight gain was calculated as the following:

$$
\text { BWG }(\mathrm{g})=\text { Final Body Weight }(\mathrm{g})-\text { Initial Body Weight }(\mathrm{g})
$$

\section{Evaluation of serum lipid profile}

Total Cholesterol (TC), Triglycerides (TG), and High-density lipoproteins (HDL) in serum were analyzed using commercial kits from Spin react in the semi-auto analyzer (Rx Monza, Randox, U.K). The values of total cholesterol, triglycerides, and HDL are expressed as mg/dl.

\section{Liver marker enzymes}

Hepatic marker enzymes such as Alkaline Phosphatase (ALP), Aspartate Transaminase (AST) and Alanine Transaminase (ALT)) in serum were analyzed using commercial kits from Spinreact and Randox in the semi-auto analyzer (Rx Monza, Randox, UK).

\section{Determination of lipid peroxidation (LPO)}

LPO was evaluated in the tissue homogenate samples. To evaluate the level of LPO, the mean concentration of malondialdehyde (MDA) was assayed in the form of thiobarbituric acid-reacting substances (TBARS) with the method described by Ohkawa et al. [25].

\section{Enzymatic antioxidant activity}

The activity of enzymes in the antioxidant system was evaluated in the tissue homogenate samples following previously reported methods. Catalase (CAT) activity was determined using the method of Sinha et al. [30] and expressed as U/mg protein ( $\mu \mathrm{mol}$ of H2O2 consumed minmg protein). Superoxide dismutase (SOD) activity was determined using the method of Marklund S, Marklund G. [20] and expressed as U/mg protein. Glutathione peroxidase (GPx) was determined as described by Rotruck et al. [29] and expressed in terms of $\mu \mathrm{g}$ of reduced glutathione (GSH) consumedminimg protein. 


\section{Non-enzymatic antioxidant levels}

The levels of non-enzymatic antioxidants in liver tissue homogenate samples were determined by previously reported methods. The GSH content was estimated by the method of Moron et al. [21]. Ascorbate (vitamin C) was measured using the method of Omaye et al. [26]. $\alpha$-tocopherol (vitamin E) was estimated by the method of Desai et al. [7]. The results are expressed as $\mu$ gmg protein.

\section{Enzyme-Linked Immunosorbent Assay (ELISA)}

The levels of Ox-DL in infected cell passage no 21cell line (IC-21) and CRP in serum were measured using ELISA as described by Crowther et al. [4]. High affinity 96 well microtitre plates were coated with serum diluted in bicarbonate buffer, incubated overnight at $4{ }^{\circ} \mathrm{C}$. Plates were washed thrice with $0.15 \mathrm{M}$ phosphate buffered saline ( $\mathrm{pH}$ 7.4) and then blocked for 1 hour at room temperature with $200 \mu \mathrm{l} /$ well PBS containing1\% BSA. The plates were washed twice with PBS before the addition of suitably diluted primary antibody and incubated overnight at $4^{\circ} \mathrm{C}$, then washed four times with PBS. Afterwards, $100 \mu 1$ of horseradish peroxidase-conjugated secondary antibody diluted in blocking buffer was added and incubated for 1 hour at room temperature. After washing with PBS twice, immune reactivity was visualized by the addition of $50 \mu \mathrm{l} /$ well 2, 2'-azino-bi's (3-ethylbenzothiazoline-6-sulphonic acid) (ABTS) substrate for 15 min at room temperature. The reaction was stopped by the addition of $50 \mu \mathrm{l} /$ well of $2 \mathrm{M} \mathrm{H}_{2} \mathrm{SO}_{4}$, and the optical densities were determined at $450 \mathrm{~nm}$ using a plate ELISA reader (BioTek, USA). The levels are expressed as $\mathrm{pg} / \mathrm{ml}$.

\section{Histopathological studies}

Histology of liver was studied using hematoxylin and eosin ( $\mathrm{H} \& \mathrm{E})$. The portion of liver tissues was fixed in $10 \%$ buffered formalin. The washed tissues were dehydrated in the descending grades of isopropanol and finally cleared in xylene. The tissues were then embedded in molten paraffin wax. Sections were cut at $5 \mu \mathrm{m}$ thickness and stained with H\&E. The sections were then viewed under the light microscope (Nikon microscope ECLIPSE E400, Japan) for histopathological changes.

\section{Western blot analysis}

The tissue homogenate was prepared in 50-100 $\mathrm{ml}$ of lysis buffer (with protease inhibitors), centrifuged and the supernatant was collected. Total tissue extracts containing 50-100 $\mu \mathrm{g}$ of protein samples were prepared in Sodium Dodecyl Sulphate (SDS) sample buffer (SigmaAldrich) and were separated by SDS-electrophoresis on $10 \%-12 \%$ polyacrylamide gels, further transferred to polyvinylidene difluoride membranes prior to immunodetection and subjected to western blot analysis. The antibodies against NF- $\mathrm{B}(\mathrm{NF}-\kappa \mathrm{B}$; rabbit polyclonal; 1:1,000), IL6 (IL6; rabbit polyclonal; 1:1,000), and VCAM1 (VCAM1; goat anti-rabbit; 1:1,000) was purchased from Santa Cruz Biotechnology and was used to detect protein levels in the liver tissues. To verify the uniformity of protein load and transfer efficiency across the test samples, membranes were reproved with ßeta-actin (Cell Signaling Technology, 1:1000 dilution). Immuno-reactive bands were developed by Immobilon Western-Chemiluminescent HRP substrate (Millipore Corporation, Billerica, USA), visualized using an enhanced chemiluminescence system (Chemi-Doc, BioRad, USA) and presented in comparison to ßetaactin expression. 
Statistical analysis

Statistical analysis was performed using SPSS 19 package. Values represent Mean \pm SEM for six rats in each group and the significance of the difference between mean values was determined by one-way analysis of variance (ANOVA) followed by Tukey's multiple comparison tests.

\section{RESULTS}

\section{Body weight gain (BWG)}

Table 1 displays the impact of AG on body weight gain in HCD fed aged rats in comparison to young and aged experimental rats. The initial body weights of young rats were around 128 grams, while that of aging rats was 250 to 260 grams. All the experimental groups had an incremental weight gain at the end of the experimental period. On feeding HCD, there was a significant increase $(\mathrm{p}<0.05)$ in the body weight by almost two-fold when compared to their respective control. AG or AV supplementation in aged rats demonstrated a lesser body weight gain, even when fed with high cholesterol diet. Similarly, the relative organ weight of the rats also demonstrated a significant increase $(\mathrm{p}<0.05)$ upon HCD and AG supplementation was able to bring down the levels near normal as compared to AV.

Table 1. Effect of AG vs AV on body weight gain in HCD fed aged experimental rats

\begin{tabular}{lcccccc} 
Parameters & Group 1 & Group 2 & Group 3 & Group 4 & Group 5 & Group 6 \\
\hline $\begin{array}{l}\text { Initial body } \\
\text { weight* (g) }\end{array}$ & $128 \pm 12$ & $257 \pm 18^{\mathrm{a}}$ & $254 \pm 21^{\mathrm{b}}$ & $253 \pm 17^{\mathrm{c}}$ & $256 \pm 19^{\mathrm{c}}$ & $250 \pm 17^{\mathrm{b}}$ \\
$\begin{array}{l}\text { Final body weight } \\
*(\text { g) }\end{array}$ & $173 \pm 12$ & $334 \pm 27^{\mathrm{a}}$ & $411 \pm 30^{\mathrm{b}}$ & $360 \pm 38^{\mathrm{c}}$ & $372 \pm 35^{\mathrm{c}}$ & $321 \pm 26^{\mathrm{b}}$ \\
$\begin{array}{l}\text { Body weight } \\
\text { gain*(g) }\end{array}$ & $45 \pm 4$ & $76 \pm 7^{\mathrm{a}}$ & $156 \pm 12^{\mathrm{b}}$ & $107 \pm 7^{\mathrm{c}}$ & $115 \pm 8^{\mathrm{c}}$ & $70 \pm 4^{\mathrm{b}}$ \\
\hline & & & LIVER & & & \\
\hline $\begin{array}{l}\text { Relative } \\
\text { weight* }(\mathrm{g})\end{array}$ & 0.148 & $3.0 \pm 0.237^{\mathrm{a}}$ & $4.15 \pm 0.352^{\mathrm{b}}$ & $3.31 \pm 0.29^{\mathrm{c}}$ & $3.57 \pm 0.32^{\mathrm{c}}$ & $3.02 \pm 0.22^{\mathrm{b}}$ \\
& $2.12 \pm$ & & & & &
\end{tabular}

Values are expressed as grams. Body weight gain (g) was calculated as the following: The difference between initial body weight $(\mathrm{g})$ and final body weight $(\mathrm{g})$ and relative organ weight to body weight is the ratio of absolute organ weight $(\mathrm{g})$ to body weight $(\mathrm{g})$.

Values are expressed as grams. Body weight gain (g) was calculated as the following: The difference between initial body weight $(\mathrm{g})$ and final body weight $(\mathrm{g})$. Each value represents mean \pm SEM for six rats in each group. Values are statistically significant at the level of $\mathrm{p}<0.05$, where 'a' - compared with Group 1, 'b' -compared with Group 2, 'c' - compared with Group 3; Group 1- Young control; Group 2- Aged control; Group 3- Aged HCD; Group 4- Aged HCD +AG; Group 5- Aged HCD+AV; Group 6- Aged +AG alone.

\section{AG vs AV assessment of serum lipid profile:}

The aged rats exhibited an increase in the serum TC (1.38 fold), TG (1.35 fold), LDL (3.38 fold), Very low-density lipoprotein (VLDL) (1.33fold), Atherogenic Index (2.18 fold) along with a concomitant decrease in HDL (1.61 fold) levels when compared to the young control group. 
Aged HCD fed rats exhibited a significant $(\mathrm{P}<0.05)$ increase in the serum TC $(2.29$ fold $)$, TG (2.39 fold), LDL (3.17fold), VLDL (2.3 fold), Atherogenic Index (3.68 fold) levels along with a concomitant decrease in HDL (3.61 fold), when compared to the aged control group. However, HCD fed aged rats when treated with AG [TC (60.228\%), TG (37.82\%), LDL (94.57\%), HDL (261.8\%), VLDL (37.02\%), and atherogenic index (64.69\%)] levels were brought down. On the other hand, AV treatment led to [TC (46.52\%), TG (29.05\%), LDL (66.06\%), HDL (82.68\%), VLDL (28.30\%), and atherogenic index (37.15\%)] exhibiting a significant $(\mathrm{P}<0.05)$ decrease in their serum lipid profiles except HDL levels which was increased as its good cholesterol to nearnormal levels. Significantly, the AG by itself treated aged rats exhibited an increase in HDL (1.27 fold) along with a decrease in TC (1.16 fold), TG (1.25 fold), LDL (1.44 fold), VLDL (1.26 fold) and atherogenic index (2.44 fold) compared to that of age control group rats (Table 2.). Similarly, the levels of $\mathrm{TC}$ and $\mathrm{TG}$ were significantly increased $(\mathrm{P}<0.05)$ in liver tissue samples of aging and aged HCD fed rats, while it was brought down to near normal levels by AG and AV supplementation. AG was also observed to bring down the effect of aging, as observed in group 6.

Table 2. Effect of AG vs AV on serum lipid profile in HCD fed aged experimental rats

\begin{tabular}{lllllll} 
GROUPS & GROUP 1 & GROUP 2 & GROUP 3 & GROUP 4 & GROUP 5 & GROUP 6 \\
\hline $\begin{array}{l}\text { Total } \\
\text { Cholesterol } \\
\text { Triglyceride }\end{array}$ & $160.10 \pm 11.57$ & $213.77 \pm 17.74^{\mathrm{a}}$ & $510 \pm 49.52^{\mathrm{b}}$ & $372.21 \pm 31.41^{\mathrm{c}}$ & $397.5 \pm 28.62^{\mathrm{c}}$ & $170 \pm 13.44^{\mathrm{b}}$ \\
LDL & $26.78 \pm 2.16$ & $90.57 \pm 6.76^{\mathrm{a}}$ & $287.55 \pm 25.87^{\mathrm{b}}$ & $147.78 \pm 15.57^{\mathrm{c}}$ & $173.15 \pm 12.58^{\mathrm{c}}$ & $62.88 \pm 4.99^{\mathrm{b}}$ \\
HDL & $67.40 \pm 5.27$ & $41.79 \pm 3.41^{\mathrm{a}}$ & $11.55 \pm 1.07^{\mathrm{b}}$ & $28.11 \pm 2.84^{\mathrm{c}}$ & $21.10 \pm 1.52^{\mathrm{c}}$ & $53.33 \pm 4.15^{\mathrm{b}}$ \\
VLDL & $32.02 \pm 2.59$ & $42.754 \pm 3.54^{\mathrm{a}}$ & $102 \pm 7.24^{\mathrm{b}}$ & $74.44 \pm 6.10^{\mathrm{c}}$ & $79.5 \pm 7.15^{\mathrm{c}}$ & $34 \pm 2.58^{\mathrm{b}}$ \\
$\begin{array}{l}\text { Atherogenic } \\
\text { Index }\end{array}$ & $0.016 \pm 0.001$ & $0.349 \pm 0.029^{\mathrm{a}}$ & $1.285 \pm 0.116^{\mathrm{b}}$ & $0.762 \pm 0.072^{\mathrm{c}}$ & $0.915 \pm 0.091^{\mathrm{c}}$ & $0.143 \pm 0.0135^{\mathrm{b}}$
\end{tabular}

\section{LIVER LIPIDS}

\begin{tabular}{|c|c|c|c|c|c|c|}
\hline $\begin{array}{l}\text { Total } \\
\text { Cholesterol }\end{array}$ & $6.81 \pm 0.491$ & $9.33 \pm 0.755^{\mathrm{a}}$ & $15.91 \pm 1.46^{b}$ & $13.11 \pm 0.96^{c}$ & $13.43 \pm 1.03^{c}$ & $9.21 \pm 0.67^{\mathrm{b}}$ \\
\hline Triglyceride & $9.41 \pm 0.705$ & $12.37 \pm 1.0^{\mathrm{a}}$ & $20.75 \pm 1.92^{b}$ & $15.21 \pm 1.11^{\mathrm{c}}$ & $15.55 \pm 1.10^{c}$ & $11.71 \pm 0.85^{b}$ \\
\hline
\end{tabular}

Each value represents mean \pm SEM for six rats in each group. Values are statistically significant at the level of $p<0.05$, where ' $a$ ' - compared with Group 1, 'b' -compared with Group 2, 'c' compared with Group 3; Group 1- Young control; Group 2- Aged control; Group 3- Aged HCD; Group 4- Aged HCD +AG; Group 5-Aged HCD+AV; Group 6- Aged +AG alone.

\section{$A G$ vs $A V$ on liver marker enzymes:}

The assessment of cellular integrity was carried out by determining the activities of liver marker enzymes including ALT, AST, and ALP in serum, as shown in Fig. 1. Assessment of liver marker enzymes in serum of various experimental groups dictates that the levels of these 
enzymes were elevated both by aging and HCD feeding. There was a marked increase $(\mathrm{p}<0.05)$ in the activities of these marker enzymes in HCD fed aged and normal diet fed aged rats by about 1.5-fold. In contrast, the aged HCD fed rats demonstrated an increase by about 2 folds, when compared to the young control rats. These results signify that the impact of HCD in aged rats was more predominant than that of control rats. Supplementation of AG and AV to HCD fed aged rats brought down the elevated activities of these enzymes when compared to their respected untreated counterparts. The $A G$ treated aged rats revealed decreased levels of the enzymes [ALT (1.16 fold), AST (1.18 fold), and ALP (1.34 fold)] as compared to their control counterpart.
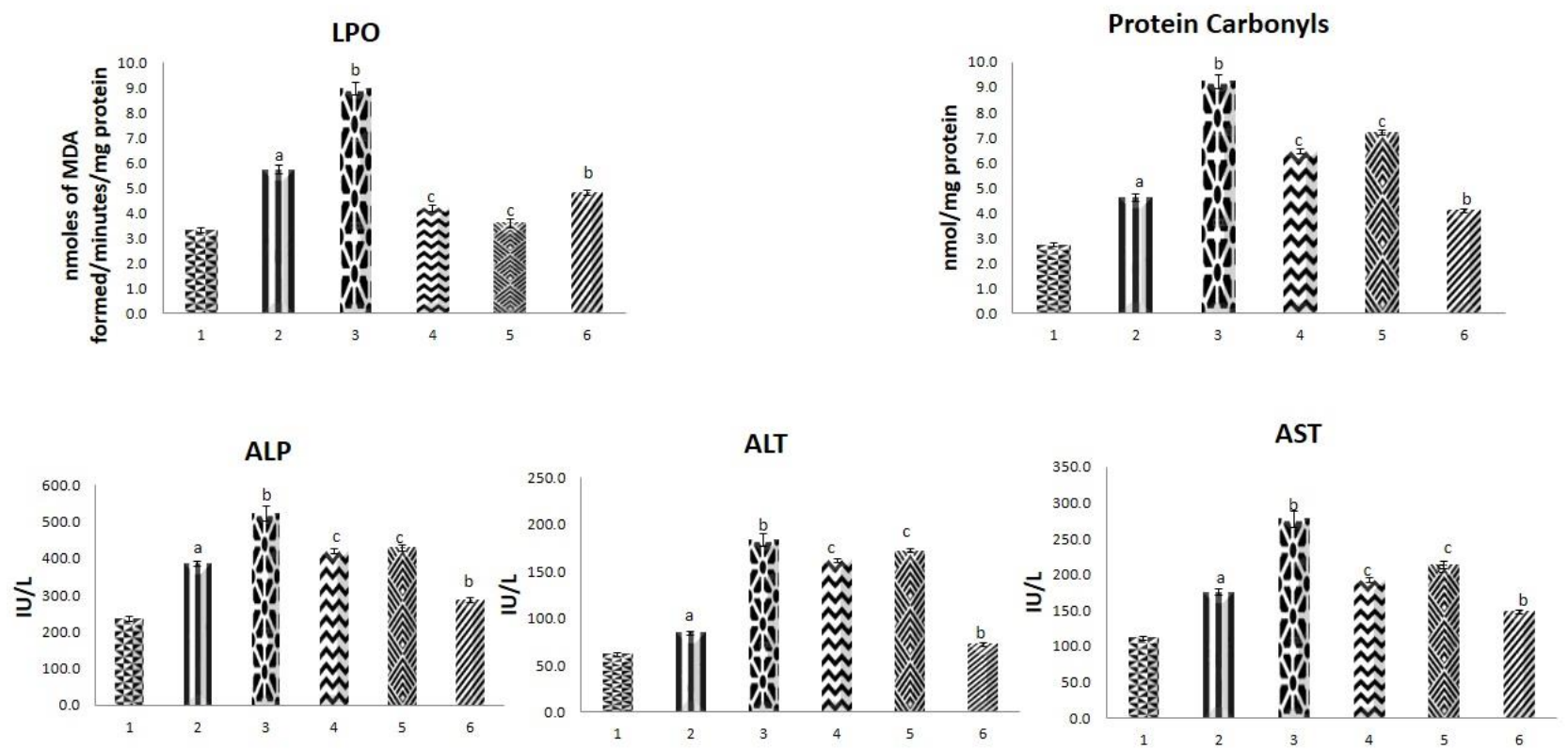

Figure 1. Effect of AG vs AV on liver markers, lipid peroxidation, and protein carbonyls of HCD fed aged liver. LPO - nmoles of MDA/mg protein; PC - nmol/mg of protein. Each bar represents mean \pm SEM for six rats in each group. Values are statistically significant at the level of $\mathrm{p}<0.05$, where ' $\mathrm{a}$ ' - compared with group 1, 'b' -compared with group 2, 'c' - compared with group 3; Group 1- Young control; Group 2- Aged control; Group 3- Aged HCD; Group 4- Aged HCD +AG; Group 5- Aged HCD+AV; Group 6- Aged +AG alone

\section{Impact of $A G$ vs $A V$ and role of $L P O$ in the liver tissue damage:}

LPO was determined by the mean concentration of MDA assayed in the form of TBARS. The liver tissue samples from aged control rats demonstrated an increase ( 1.75 fold) in the levels of MDA compared to the young control group; in contrast, HCD fed rats exhibited a significant $(\mathrm{P}<0.05)$ increase $(1.56$ fold) in the levels of MDA compared to the aged control (Fig. 1). On the other hand, in the HCD+AG group, LPO was significantly $(\mathrm{P}<0.05)$ brought down to 2.49 fold in the liver tissue samples. Similarly, the HC+AV group demonstrated a marked decrease of 2.13 fold. Additionally, when the aged rats were treated with AG, they exhibited notably improved protection of 1.18 fold against LPO compared to the aged control rats.

\section{Effect of $A G$ vs $A V$ on enzymatic antioxidant activity and non-enzymatic antioxidants levels:}

Figure (2) demonstrates the levels of enzymatic and non-enzymatic antioxidant. A prominent decrease in the levels of the antioxidants in the aged control rats compared to the young control rats was observed. Additionally, a significant $(\mathrm{P}<0.05)$ decrease in the mean activity of the 
enzymatic antioxidants CAT (37.16\%), SOD (83.21\% and GPx (16.1\%) were observed in liver tissue of HCD fed rats when compared to control rats, while $\mathrm{HCD}+\mathrm{AG}$ treated rats exhibited improved CAT (1.62 fold), SOD (2.41 fold), and GPx (2.16 fold) antioxidant activities. Similarly, there was decrease in the levels of the non-enzymatic antioxidants GSH (1.40 fold), ascorbate (1.69 fold), and $\alpha$-tocopherol (36.91 fold) in aged control rats when compared to young control rats. The mean levels of SOD, CAT, GSH, GPx, Ascorbate, and $\alpha$-tocopherol in the liver tissue samples of $\mathrm{AG}$ alone treated aged rats demonstrated a significant difference when compared to the aged control group of rats establishing the role of AG in reversing, age effected antioxidant profile to a significant extent.
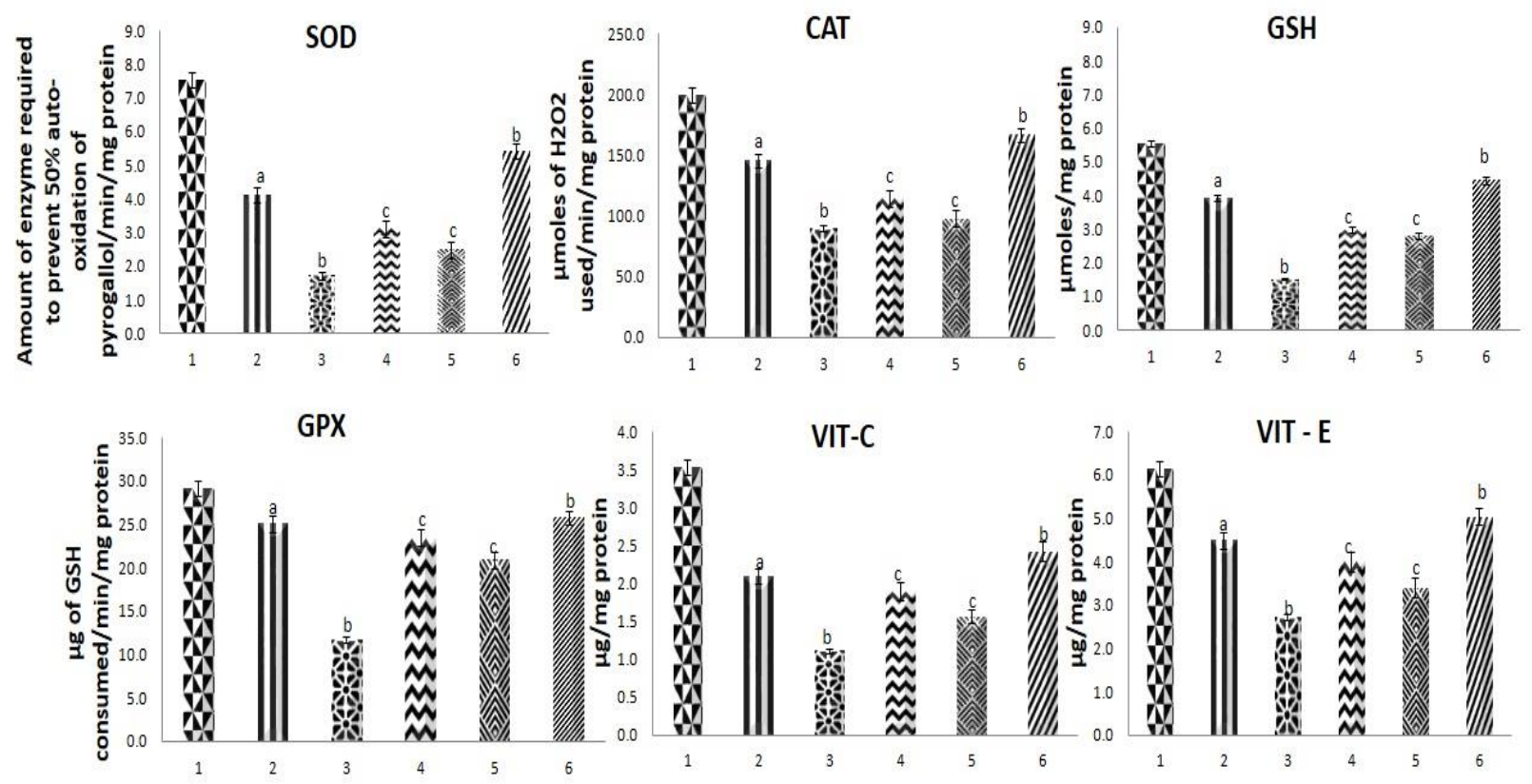

Figure 2. Beneficial role of AG vs AV on Enzymic and Non-Enzymic antioxidants in liver of aged rats fed with HCD. SOD - Amount of enzyme required to prevent 50\% auto-oxidation of pyrogallol $/ \mathrm{min} / \mathrm{mg}$ protein; CAT - $\mu \mathrm{mol}$ hydrogen peroxide degraded $/ \mathrm{min} / \mathrm{mg}$ of protein; GPx - $\mu \mathrm{g}$ of GSH consumed $/ \mathrm{min} / \mathrm{mg}$ protein; GSH - nmoles $/ \mathrm{mg}$ protein; VIT E- $\mu$ gram $/ \mathrm{mg}$ protein. VIT C- $\mu$ gram $/ \mathrm{mg}$ protein. Each bar represents mean \pm SEM for six rats in each group. Values are statistically significant at the level of $p<0.05$, where ' $a$ ' - compared with group 1, 'b' -compared with group 2, 'c' compared with group 3; Group 1- Young control; Group 2- Aged control; Group 3- Aged HCD; Group 4Aged HCD +AG; Group 5- Aged HCD+AV; Group 6- Aged +AG alone

\section{Effect of $A G$ vs $A V$ on histopathological changes of HCD fed liver:}

The abnormal fatty changes in hepatic tissue histology in aged and HCD fed aged rats along with treatment of AG were presented in Figure. 3. Hematoxylin-Eosin stained liver sections from young control rats demonstrated chords of normal hepatocytes with no pathological features (Group 1), while normal diet fed aged rats (Group 2) revealed mild fatty changes and fewer signs of liver damage. Hepatic tissue from HCD fed aged rats (Group 3) revealed increased microvesicular and macrovesicular steatosis, which appeared on the lobules and was more evident. Aged HCD fed rat liver also demonstrated periportal infiltration by inflammatory cells, 
ballooning degeneration, and signs of liver damage originating from the intralobular infiltration of mononuclear cells such as macrophages, lymphocytes, and hepatocytes necrosis. No fibrotic changes were observed in the hepatic tissue of $\mathrm{HCD}+\mathrm{AG}$ treated aged rats (Group 4). Moreover, AV treated HCD fed aged (Group 5) and aged control (Group 2) rats demonstrated occasional hepatocytes with microvesicular steatosis, reduced fatty changes and liver damage, reduced macrophage infiltration and reduced necrosis when compared to untreated groups. (Group 6) rats appeared normal.

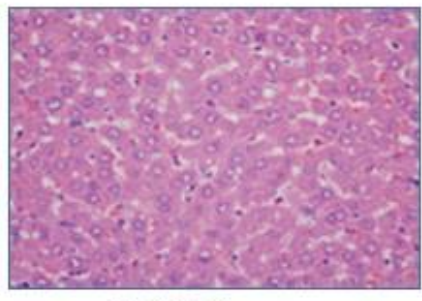

GROUP 1

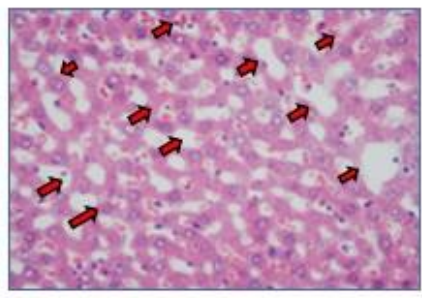

GROUP 3

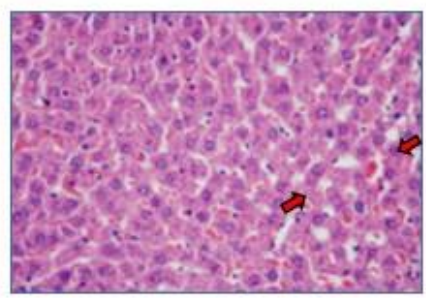

GROUP 5

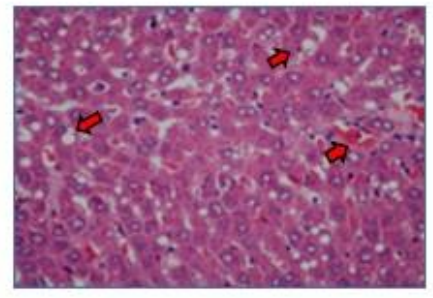

GROUP 2

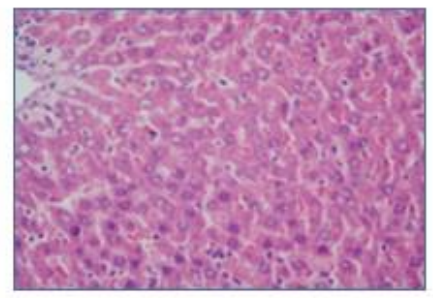

GROUP 4

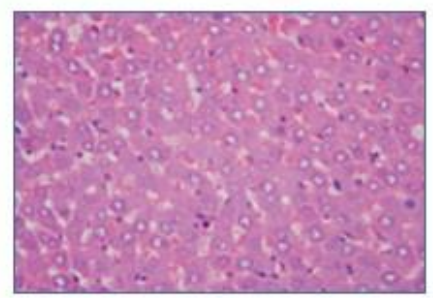

GROUP 6

Figure 3. Histopathological sections of liver of various experimental groups fed with HCD along with or without AG/AV [20X]. Light Microscopic analysis of the pathological H\&E stained liver sections of rats (20X) from; Group 1- Young control; Group 2- Aged control; Group 3- Aged HCD; Group 4- Aged HCD +AG; Group 5- Aged HCD+ AV; Group 6- Aged +AG alone: In the young control group (1) Hematoxylin-Eosin stained liver sections from young control rats had normal hepatocytes with no pathological features: In the aged control group (2) standard diet fed aged rats demonstrated mild fatty changes: In the aged+ HCD (3) hepatic tissue from HCD fed aged rats revealed increased micro vesicular and macro vesicular steatosis. In the aged HCD+ AG (4) rats demonstrated reduced fatty changes and liver damage, reduced macrophage infiltration and reduced necrosis when compared to untreated groups; In the aged + HCD+ AV (5) On treatment with AV no demonstrated fibrotic changes, mild fatty changes observed in the hepatic tissue of HCD fed aged rats: In the aged+ AG (6) rats revealed more or less similar hepatic muscle integrity as in control group 2.

\section{$A G$ vs $A V$; Effect on protein expression profile:}

We detected the key expression profiles of proteins in serum and cell line by Enzyme-Linked Immunosorbent Assay (ELISA). Significantly, aging alone demonstrated a higher expression 
profile [CRP-2.57 fold] when compared to the young control. And aging +HCD increased the CRP levels to 1.6 fold compared to aged control, while the treatment group of AG demonstrated marked decrease by 1.38 fold; similarly, AV treatment had similar outcome and AG alone treatment revealed no marked difference in levels of CRP. The levels of Ox-LDL induced damage in IC21 cell line was observed in different groups the Ox-LDL introduction to IC 21 demonstrated increases of 3.6 fold in comparison to control group; while Ox-LDL+AG treated rats exhibited significant $(\mathrm{P}<0.05)$ decrease in protein levels of Ox-LDL by 2.2 fold, Ox-LDL when treated to an IC-21 cell line is able to show difference at just three-hour interval by 2.8 fold; and maximum damage to these cell lines is when it's treated with a six-hour interval to about $84 \%$ [Fig 4.]. AG treatment alone on IC-21 cell line also demonstrated the marked difference compared to the control group. Similarly, at the tissue level the expression of NF- $\kappa \mathrm{B}$ [Fig 5.)]; IL6 [Fig. 4)]; and VCAM1 [Fig. 6] demonstrated a significant $(\mathrm{P}<0.05)$ increase in aged and aged + HCD groups by [NF- $\beta-59.77 \%$, IL6-54.53\%, and VCAM1-55.16\%] compared to the young control; and on treatment with AG and AV the levels of expression were markedly improved to a significant extent. It was also observed that AG by itself on the aged rats could revert the effect of aging.
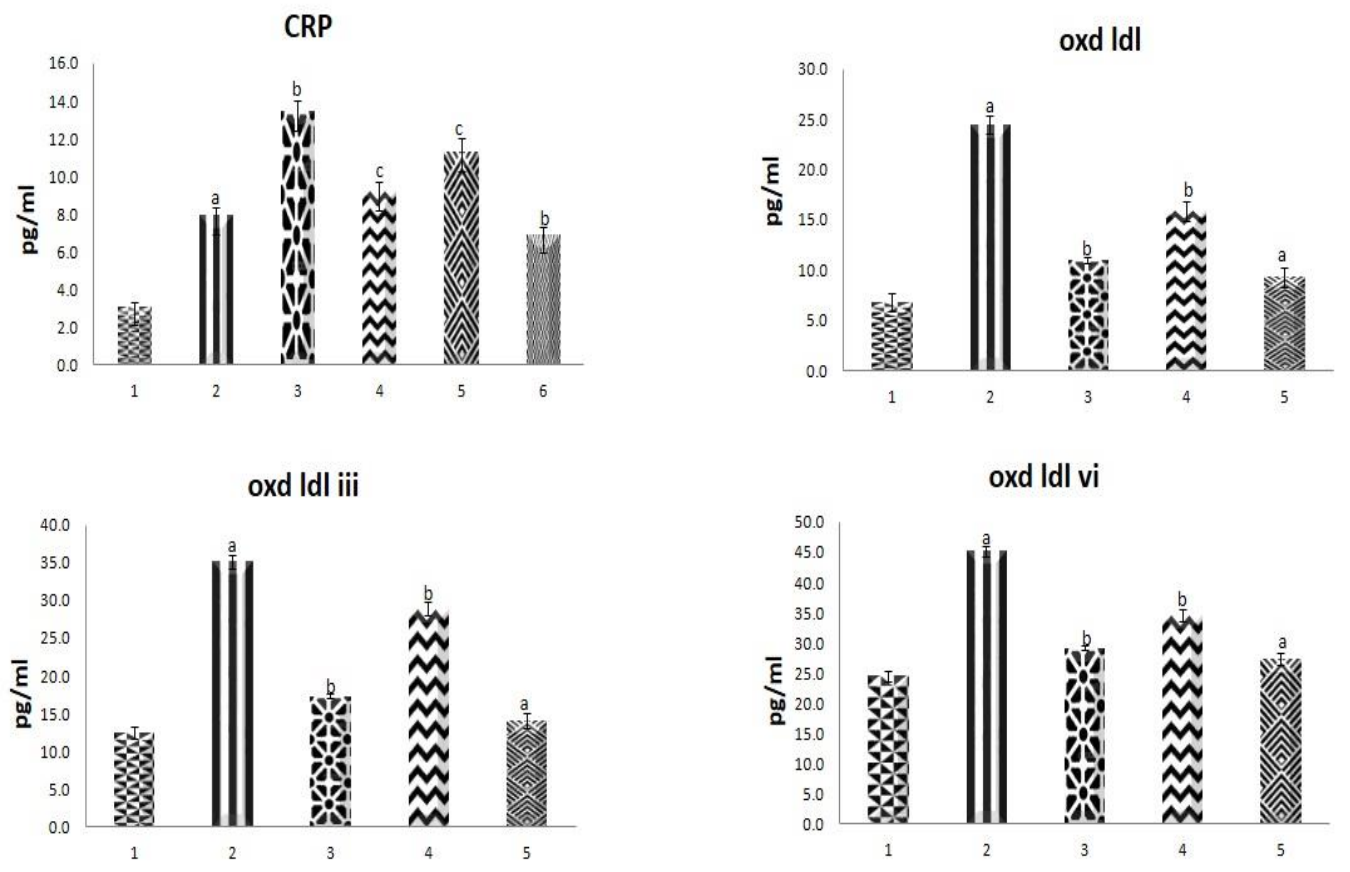

Figure 4. Beneficial role of $A G$ vs $A V$ on serum $C R P$ in $\mathrm{HCD}$ fed aged experimental rats and Effect of AG vs AV on IC-21 cell line for Ox- LDL treatment. Each bar represents mean \pm SEM for six rats in each group. Values are statistically significant at the level of $p<0.05$, where ' $a$ ' - compared with group 1, 'b' -compared with group 2, 'c' - compared with group 3; Group 1- Young control; Group 2- Aged control; Group 3- Aged HCD; Group 4- Aged HCD +AG; Group 5- Aged HCD+AV; Group 6Aged + AG alone; Each bar represents mean \pm SEM for three samples of cells in each group. Values are statistically significant at the level of $\mathrm{p}<0.05$, where ' $\mathrm{a}$ ' - compared with group 1 , ' $\mathrm{b}$ ' -compared with group 2, 'c' - compared with group 3; Group 1- Native LDL; Group 2- Native LDL4; Group 3- Native

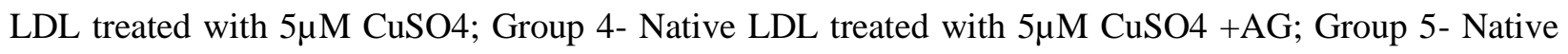
LDL treated with $5 \mu \mathrm{M}$ CuSO4 +AV; Group 6- Native LDL+AG alone 

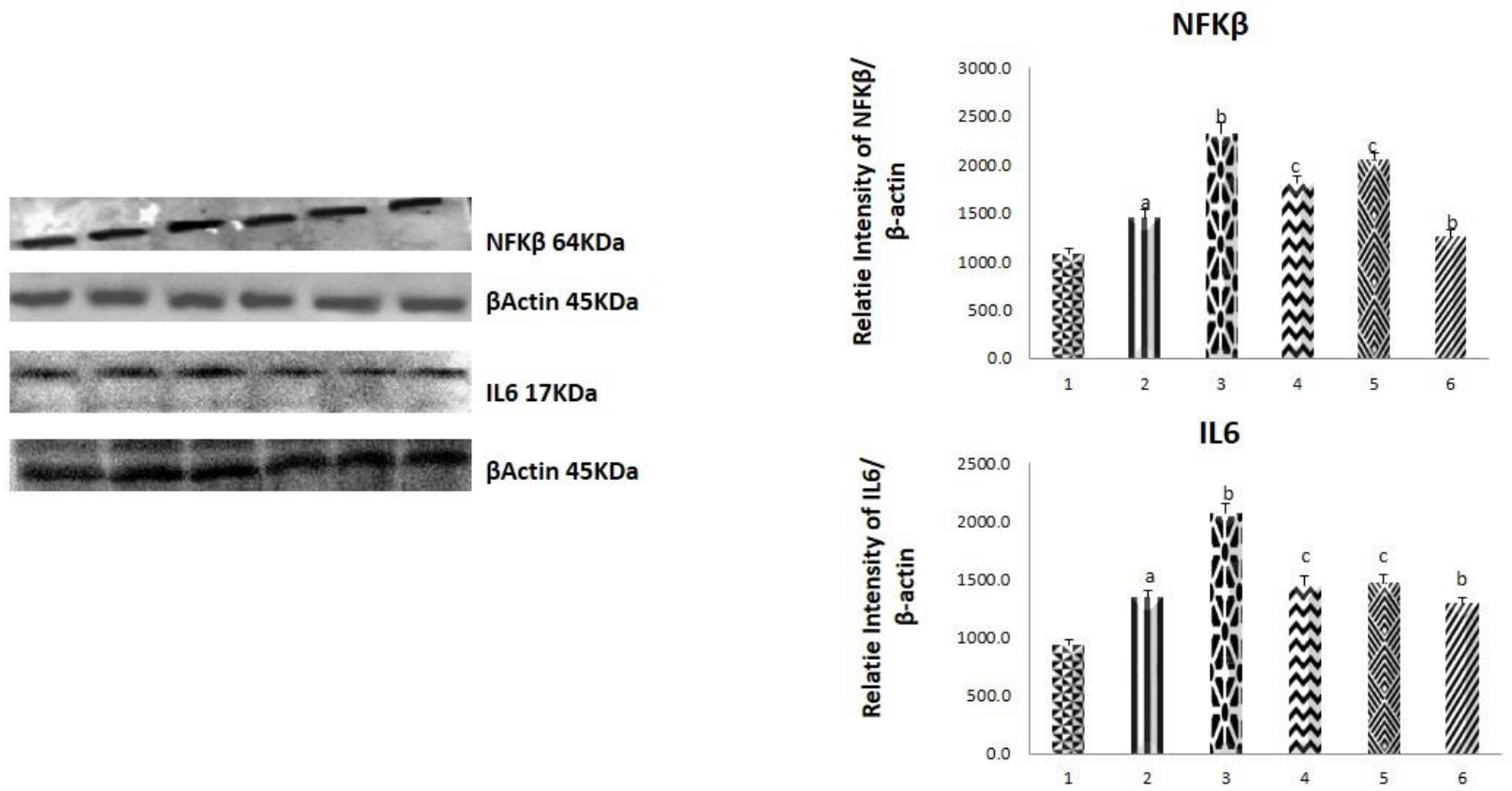

Figure 5. Effect of AG vs AV on protein expression of NFK $\beta$ and IL-6 in liver of aged rats fed with HCD. Each bar represents mean \pm SEM for six rats in each group. Values are statistically significant at the level of $\mathrm{p}<0.05$, where 'a' - compared with group 1 , 'b' -compared with group 2, 'c' compared with group 3; Group 1- Young control; Group 2- Aged control; Group 3- Aged HCD; Group 4Aged HCD +AG; Group 5- Aged HCD+AV; Group 6- Aged +AG alone
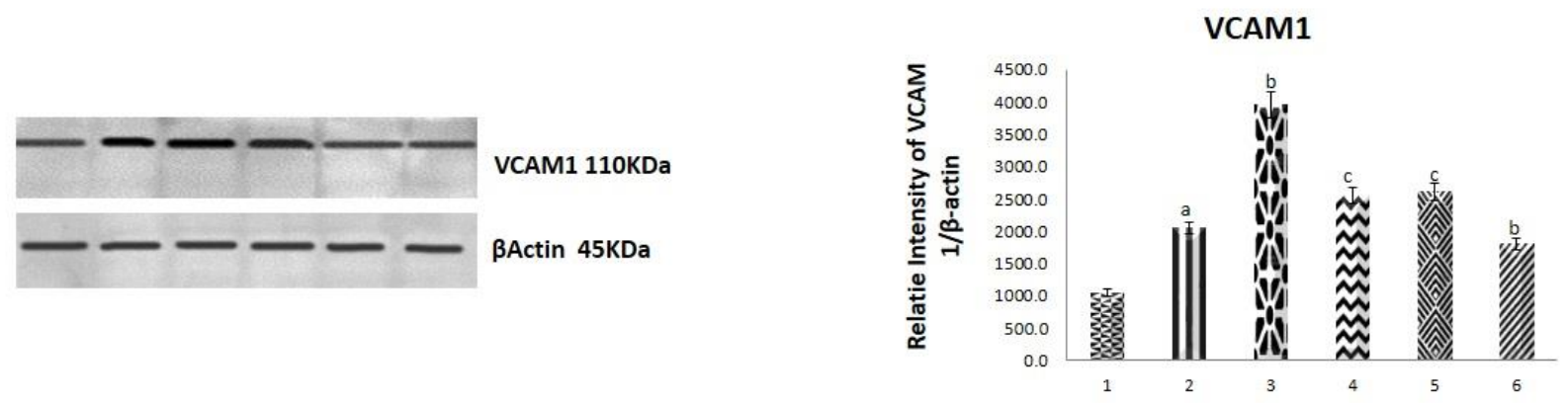

Figure 6. Effect of AG vs AV on expression of vascular cell adhesion molecule in liver of aged Rats fed with HCD. Each bar represents mean \pm SEM for six rats in each group. Values are statistically significant at the level of $\mathrm{p}<0.05$, where 'a' - compared with group 1 , 'b' -compared with group 2, 'c' compared with group 3; Group 1- Young control; Group 2- Aged control; Group 3- Aged HCD; Group 4Aged HCD +AG; Group 5- Aged HCD+AV; Group 6- Aged +AG alone

\section{DISCUSSION}

HCD consumption has dramatically increased and is suspected to be linked to metabolic disorders and chronic diseases. High-level cholesterol consumption can lead to weight gain and elevated blood lipid levels [15]. Numerous studies have demonstrated that active nutraceuticals have potential therapeutic effects on metabolic conditions induced by high cholesterol feeding. In this study, we have demonstrated for the first time that AG, a natural alkaloid amide, efficiently inhibits HCD induced body weight gain, hyperlipidaemia, and liver injury by enhancing the lipid clearance and antioxidant defense system, in addition to regulating the 
secretion of inflammatory cytokines. Numerous studies have demonstrated that diets high in cholesterol may result in elevated TG, TC, VLDL, and LDL and decreases in HDL, which are known as risk factors for cardiovascular diseases [22]. Our experimental results were consistent with earlier studies that discovered HCD fed animals developed hyperlipidaemia. Administration of AG, notably protected against the increases in serum total Cholesterol, VLDL, Triglycerides, and LDL and defended the system by increasing the levels of HDL in aged hyperlipidaemic rats. To find out the extent of protection given by AG on HCD mediated lipid derangements and hepatocellular damage; we investigated the levels of Ox- LDL, CRP, AST, ALT, and ALP in aged and aged HCD fed rats. Surprisingly, these levels were brought down significantly upon AG supplementation when compared to AV. Literature survey depicts that anti-atherogenic HDL, has pleiotropic biological functions, such as promoting cholesterol efflux, stimulating reverse lipid transport, preventing LDL oxidation, scavenging toxic phospholipids, and exhibiting anti-inflammatory activity [10]. Thus, the HDL boosting activity of AG is reflected in the lowered levels of these markers. Additionally, oxidation of LDL and their further uptake by macrophages is known to result in the foam cell formation a critical step in the initiation of atherosclerotic events. In the current context, we have observed the effect of AG vs. AV on OxLDL induced damage in the macrophage cell line IC-21. The importance of this macrophage survival pathway in atherogenesis and liver steatosis was recently elucidated [29]. Accordingly, AG could effectively prevent this Ox-LDL induced macrophage cell damage.

Histopathological observations of liver sections demonstrated that HCD-fed rats demonstrated severe lipid droplets deposition, vacuolation, and nuclear fragmentation, while AG supplementation protected liver tissue from the HCD-induced fatty liver and hepatic damage as compared to AV. Furthermore, the biochemical analyses of ALT, ALP, and AST activities of HCD-fed aged rats were consistent with the findings of the liver histopathological change. In our study, the levels of ALT, AST, and ALP were obviously enhanced by the HCD diet, and AG treatment significantly mitigated this alteration (Figure. 1). These results suggested for the first time that "AG" is able to lessen the hepatic injury induced by HCD. Oxidative stress is involved in the pathogenesis of many chronic progressive diseases. Plenty of evidence has indicated that dietary cholesterol is able to inactivate antioxidant defense and to elevate the production of free radicals [34]. Therefore, we demonstrated the effects of AG on the typical indices of oxidative stress, including antioxidant enzyme activities (CAT, SOD, GSH-Px, and Vitamin C\&E) and lipid peroxide in the liver. It was observed that the activities of these antioxidants were depressed in the HCD fed rats. AG supplementation enhanced the antioxidant enzyme activities in the liver of these rats (Figure. 2). MDA, a characteristic indicator of oxidative injury was increased in HCD-fed aged rats and found that the administration of AG effectively reduced MDA levels in liver tissue (Figure. 1).

Overall, this investigation pointed out that AG could inhibit HCD-induced hepatic oxidative injury. Numerous studies have shown that HC enriched diets possibly leads to a fatty liver, and the fatty liver is closely linked to increased secretion of pro-inflammatory cytokines, including NFK $\beta$, IL-6, and VCAM1. It has been reported that the HCD diet could significantly enhance the levels of NFK $\beta$ and IL-6 in Sprague-Dawley rats [9]. Similar effects were observed in the present study while the results revealed that rats fed with HCD demonstrated a significant increase in the levels of pro-inflammatory cytokines. Among the cell adhesion molecules, ICAM-1 and VCAM-1 have been considered to be basic and important molecules for the 
macrophages to infiltrate into the cells during the development of liver damage [1]. Studies done by Lalor et al. [13] demonstrated how VCAM-1 has been shown to mediate monocyte capture and adhesion via $\alpha 4$ integrins in vivo [14]. Furthermore, In vitro studies demonstrate that monocyte adhesion to hepatic sinusoids is inhibited by blocking VCAM-1 [1], thereby playing a role in macrophage infiltration. In the present study, the protein expression of VCAM-1 levels was discovered to be significantly up-regulated in aged rats. These reports are in accordance with the studies done by Zou et al. and Culmer et al. [35; 6], who have stated that aging results in a significant increase in soluble cell adhesion molecules. As proposed by Zou et al. [35], increased oxidative stress may be one of the factors responsible for the elevated expression of cell adhesion molecules in aged rats in the present study. However, this increase was markedly downregulated by AG treatment. The elevated levels of NFK- $\beta$ and IL- 6 also suggest that the HCD diet may contribute to fatty liver, at least in part by up-regulating hepatic inflammation. Moreover, the expression profile of proteins involved in various stages of atherosclerosis such as IL-6, CRP, VCAM-1, and selectins is basically altered during aging [5;8]. Thus, studies using aged rats will be more pertinent than the young ones. Hepatic events including the release of inflammatory markers like CRP are shown to modulate atherogenesis, so the close association has been established between hepatic steatosis and cardiovascular disease. However, the events that trigger hepatic steatosis have been kept in dark for prognosis or diagnosis of cardiovascular disease. It was observed in the current study that AG could abrogate the inflammatory responses in the liver of aging rats, which serve as evidence of how AG supplementation can be beneficial in associated metabolic derangements and inflammatory events in the absence of HCD intake. In conclusion, this study clearly demonstrates that HCD induces hyperlipidaemia, hepatic steatosis, liver oxidative injury, and hepatic inflammation. However, AG effectively prevents the development of HCD-induced hyperlipidaemia and liver injury similar to AV in many of the studied parameters and more efficiently on controlling inflammation. This finding suggests that AG could be an effective novel preventive ingredient for some chronic inflammatory diseases induced by a high-cholesterol diet like non-alcoholic fatty liver disease (NAFLD) and atherosclerosis.

\section{CONCLUSION}

Our study demonstrated that a 30-day intake of AG reduced HCD induced oxidative stress compared to AV. Additionally, the levels, oxidative stress marker, Ox-LDL, and oxidative signalling mediated pro-inflammatory cytokines were brought down near normal levels establishing AG as lipid regulator with no observed side effects. This study suggests that early intervention of "AG" during hepatic steatosis might safeguard the cardiovascular system by attenuating inflammatory events in the liver.

Authors' Contributions: All authors contributed to this study.

List of Abbreviations: AG, Aegeline; AV, Atorvastatin; HCD, High Cholesterol Diet; Ox- LDL, Oxidized low density lipoprotein; MDA, Malondialdehyde; CVD, Cardiovascular disease; NAFLD, Non-alcoholic fatty liver disease; LDL, Low density lipoprotein; CRP, C Reactive protein; IL6, Interleukin 6; VCAM1, Vascular Cell Adhesion Molecule. 
Competing Interests: The authors declare that they have no potential conflict of interest, including any financial, personal or other relationships, with other people or organizations.

Acknowledgements and Funding: The authors wish to acknowledge the financial support offered by UGC UPE Phase II Programme, University of Madras, and Chennai, India for conducting this study.

\section{REFERENCES}

1. Adams DH, Ju C, Ramaiah SK, Uetrecht J, Jaeschke H: Mechanisms of immunemediated liver injury. Toxicol Sci 2010, 115(2):307-21.

2. Crowther, John.R. ELISA: theory and practice. Springer Science \& Business Media 1995: 42

3. Csiszar A, Wang M, Lakatta EG, Ungvari Z: Inflammation and endothelial dysfunction during aging: role of NF-kappaB. J Appl Physiol 2008, 105(4):1333-41.

4. Culmer DL, Diaz JA, Hawley AE, Jackson TO, Shuster KA, Sigler RE, Wakefield TW, et al.: Circulating and vein wall P-selectin promote venous thrombogenesis during aging in a rodent model. Thromb Res 2013, 131(1):42-8.

5. Desai, ID: Vitamin E analysis methods for animal tissues. Methods Enzymol 1984, 105: 138-147.

6. Fu WJ, Haynes TE, Kohli R, Hu J, Shi W, Spencer TE, Carroll RJ, et al.: Dietary Larginine supplementation reduces fat mass in Zucker diabetic fatty rats. J Nutr 2005, 135 (4):714-21.

7. Gamboa-Gomez, CI, Rocha-Guzman NE, Gallegos-Infante JA, Moreno-Jimenez MR, Vázquez-Cabral BD, González-Laredo RF: Plants with potential use on obesity and its complications. EXCLI journal 2015, 14: 809.

8. García-Lafuente, A, Guillamón E, Villares A, Rostagno MA, Martínez JA: Flavonoids as anti-inflammatory agents: implications in cancer and cardiovascular disease. Inflammation Research 2009, 58(9): 537-552.

9. Lalor PF, Edwards S, McNab G, Salmi M, Jalkanen S, Adams DH: Vascular adhesion protein-1 mediates adhesion and transmigration of lymphocytes on human hepatic endothelial cells. The Journal of Immunology 2002, 169(2): 983-992.

10. Lee WY, Kubes P: Leukocyte adhesion in the liver: distinct adhesion paradigm from other organs. Journal of hepatology 2008, 48(3): 504-512.

11. Li M, Diao Y, Liu Y, Huang H, Li Y, Tan P, Liang H, et al.: Chronic Moderate Alcohol Intakes Accelerate SR-B1 Mediated Reverse Cholesterol Transport. Scientific reports 2016, 6: 33032.

12. Lowry OH, Rosebrough NJ, Farr AL, Randall RJ: Protein measurement with the Folin phenol reagent. J biol Chem 1951; 1: 193(1):265-75.

13. Maity P, Hansda D, Bandyopadhyay U, Mishra DK: Biological activities of crude extracts and chemical constituents of Bael, Aegle marmelos (L.) Corr. Indian journal of Experimental Biology 2009, 47: 849-861.

14. Marklund S, Marklund G: Involvement of the superoxide anion radical in the autoxidation of pyrogallol and a convenient assay for superoxide dismutase. European journal of biochemistry 1974, 1: 47(3):469-74. 
15. Moron MS, Depierre JW, Mannervik B: Levels of glutathione, glutathione reductase and glutathione S-transferase activities in rat lung and liver. Biochim Biophys Acta 1979, 582: 67-78.

16. Müller-Wieland D, Leiter LA, Cariou B, Letierce A, Colhoun HM, Prato S, Henry RR, et al: Design and rationale of the ODYSSEY DM-DYSLIPIDEMIA trial: lipidlowering efficacy and safety of alirocumab in individuals with type 2 diabetes and mixed dyslipidaemia at high cardiovascular risk. Cardiovascular diabetology 2017, 16(1): 70 .

17. Ohkawa H, Ohishi N, Yagi K: Assay for lipid peroxides in animal tissues by thiobarbituric acid reaction. Analytical biochemistry 1979, 1: 95(2):351-8.

18. Omaye ST, Turnbull JD, Sauberlich HE: Selected methods for the determination of ascorbic acid in animal cells, tissues, and fluids. Methods Enzymol 1979, 62: 3-11.

19. Paritha IA, Devi CS: Effect of $\alpha$-tocopherol on isoproterenol-induced changes in lipid and lipoprotein profile in rats. Indian J Pharmacol 1997, 29:399-404.

20. Riazy, M, Chen, JH, Yamamato Y, Yamamato H, Duronio V, Steinbrecher UP: OxLDL-mediated survival of macrophages does not require LDL internalization or signalling by major pattern recognition receptors. Biochemistry and Cell Biology 2011, 89(4): 387-395.

21. Rotruck JT, Pope AL, Ganther HE, Swanson AB, Hafeman DG, Hoekstra WG: Selenium: biochemical role as a component of glutathione peroxidase. Science 1973, 179: 588-590.

22. Sinha AK: Colorimetric assay of catalase. Analytical biochemistry 1972, 1: 47(2):38994.

23. Senthil Kumaran V, Arulmathi K, Srividhya R, Kalaiselvi P: Repletion of antioxidant status by EGCG and retardation of oxidative damage induced macromolecular anomalies in aged rats. Exp Gerontol 2008; 43(3):176-83.

24. Steinbrecher UP, Parthasarathy S, Leake DS, Witztum JL, Steinberg D: Modification of low density lipoprotein by endothelial cells involves lipid peroxidation and degradation of low density lipoprotein phospholipids. Proceedings of the National Academy of Sciences 1984, 81(12):3883-3887.

25. Vijaya C, Ramanathan M, Suresh B: Lipid lowering activity of ethanolic extract of leaves of Aegle marmelos (Linn.) in hyperlipidaemic models of Wistar albino rats. Indian Journal of Experimental Biology 2009, 47:182-185.

26. Yamamoto A, Sudoh, Endo A: Therapeutic effects of MI-236-B in primary hypercholesterolemia. Atherosclerosis 1984, 35:259-66.

27. Yuan L, Han X, Li W, Ren D, Yang X: Isoorientin prevents hyperlipidemia and liver injury by regulating lipid metabolism, antioxidant capability, and inflammatory cytokine release in high-fructose-fed mice. Journal of agricultural and food chemistry 2016, 64(13): 2682-2689.

28. Zou Y, Jung KJ, Kim JW, Yu BP, Chung HY: Alteration of soluble adhesion molecules during aging and their modulation by calorie restriction. FASEB J 2004, $18(2): 320-2$. 